
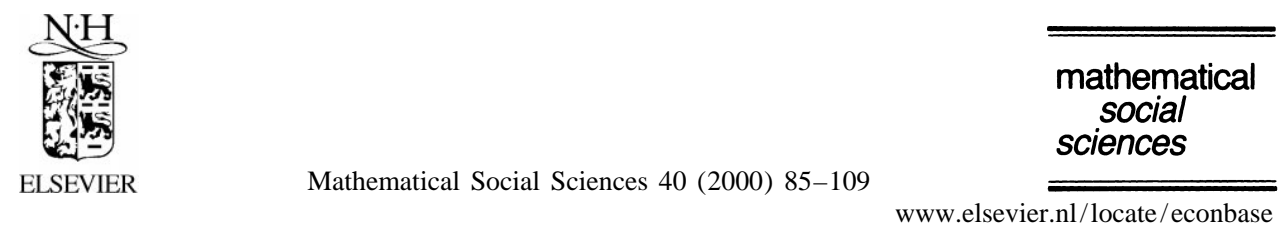

\title{
Choosing from a weighted tournament ${ }^{1}$
}

\author{
Philippe De Donder ${ }^{\mathrm{a}, *}$, Michel Le Breton ${ }^{\mathrm{b}}$, Michel Truchon ${ }^{\mathrm{c}}$ \\ ${ }^{a}$ GREMAQ, Université des Sciences Sociales, F-31000 Toulouse, France \\ ${ }^{\mathrm{b}}$ CORE, 1348 Louvain-La-Neuve, Belgium \\ ${ }^{\circ}$ CRÉFA and Département d'économique, Université Laval, Québec, Canada G1K 7P4
}

Received September 1998; received in revised form February 1999; accepted July 1999

\begin{abstract}
A voting situation, in which voters are asked to rank all candidates pair by pair, induces a tournament and a weighted tournament, in which the strength of the majority matters. Each of these two tournaments induces in turn a two-player zero-sum game for which different solution concepts can be found in the literature. Four social choice correspondences for voting situations based exclusively on the simple majority relation, and called $C 1$, correspond to four different solution concepts for the game induced by the corresponding tournament. They are the top cycle, the uncovered set, the minimal covering set, and the bipartisan set. Taking the same solution concepts for the game induced by the corresponding weighted tournament instead of the tournament and working backward from these solution concepts to the solutions for the corresponding weighted tournament and then to the voting situation, we obtain the $C 2$ counterparts of these correspondences, i.e. correspondences that require the size of the majorities to operate. We also perform a set-theoretical comparison between the four $C 1$ correspondences, their four $C 2$ counterparts and three other $C 2$ correspondences, namely the Kemeny, the Kramer-Simpson, and the Borda rules. Given two subsets selected by two correspondences, we say whether it always belongs to, always intersects or may not intersect the other one. (C) 2000 Elsevier Science B.V. All rights reserved.
\end{abstract}

Keywords: Weighted tournament; Social choice correspondences; Set-theoretical comparison

*Corresponding author. Tel.: +33-5-6112-8603; fax: +33-5-6112-8637.

E-mail address: dedonder@cict.fr (P. De Donder)

${ }^{1}$ This article is a revised version of a paper that circulated under the title 'A set-theoretical comparison of $C 2$ social choice correspondences'. 


\section{Introduction}

\subsection{Tournaments}

A tournament is a competition in which every contestant meets every other contestant in turn. ${ }^{2}$ In some tournaments, contestants may meet each other more than once, say $q$ times. In each of the $q$ encounters, one of the opponents beats the other one or they tie. We call them $q$-weighted tournaments or simply weighted tournaments, a term that we may trace back to Moulin (1988). More generally, a (weak) tournament is defined by a complete binary relation on a finite set. A (weak) weighted tournament is defined by a non-negative number $q$ and a list of numbers or weights $n(x, y)$, such that $n(x, y)+$ $n(y, x)=q$ for all pairs $(x, y)$ of different contestants. The number $n(x, y)$ is the share of $q$ that contestant $x$ gets when opposed to $y$. Weighted tournaments may be seen as tournaments with additional information as to how strong each contestant is with respect to every other contestant.

Tournaments may arise in many contexts other than sports. Our main interest is in voting situations where voters are asked to rank all candidates pair by pair as imagined by Condorcet (1785) or, equivalently, to furnish their complete preference over the set of all candidates. In this article, we define a (voting) situation as a set of candidates together with a list of preferences over this set. A situation induces a $q$-weighted tournament on the set of candidates, where $q$ is the number of voters and $n(x, y)$ is the number of voters ranking $x$ ahead of $y$. By retaining only the majority relation, i.e. whether $n(x, y) \geq n(y, x)$, the opposite or both, we get an unweighted tournament.

In Guénoche et al. (1994), one finds 0-weighted tournaments where $n(x, y)$ represents the difference between the yield of rapeseed varieties $x$ and $y$ in a given territory. Guénoche (1995) applies similar techniques to marketing. He obtains a weighted tournament by comparing nine brands of computers on a pairwise basis according to a poll of consumers. This is not very different from voting.

\subsection{Social choice correspondences}

A question that arises naturally in voting situations and thus in tournaments is how to choose a winner. This question has been the subject of many articles and books since the celebrated controversy between Borda (1784) and Condorcet (1785). Laslier (1997) provides an illuminating synthesis of these various contributions. In the language of the theory of social choice, a rule that selects a set of candidates in any voting situation is called a social choice correspondence. Condorcet advocated selecting a contestant that defeats all others more than half of the time in the $q$ encounters. Such a candidate is called a Condorcet winner. However, Condorcet was well aware that there might be a cycle in the majority relation preventing the existence of a Condorcet winner. When this happens, some other rule must be called to the rescue to break these cycles or to select some subset of contestants. A social choice correspondence that selects exclusively the

${ }^{2}$ In sports, this is called a round robin tournament. 
Condorcet winner whenever it exists is called a Condorcet consistent social choice correspondence. Many of the social choice correspondences that have been proposed in the literature, including most of those that will be considered here, are of this sort. The above concepts can be transposed to tournaments where the social choice correspondences become solutions.

Following Fishburn (1977), we classify social choice correspondences on the basis of their data requirements. A social choice correspondence belongs to the class $C 1$ if it is based exclusively on the simple majority relation, i.e. on the outcomes of all pairwise majority comparisons. The $C 2$ social choice correspondences require more data, namely the size of the majorities or the numbers $n(x, y)$. Social choice correspondences requiring more data form the class $C 3$. We could define another class contained in $C 2$ and containing $C 1$, say the class $C 1.5$, consisting of social choice correspondences that use the numbers $n(x, y)-n(y, x)$ instead of the separate numbers $n(x, y)$ and $n(y, x)$. However, all $C 2$ social choice correspondences that have been considered in the literature and those that we shall propose in this article use only the numbers $n(x, y)-n(y, x)$. Thus Fishburn might have defined the class $C 2$ as consisting of all correspondences that are based on the numbers $n(x, y)-n(y, x)$. This is the definition that we shall adopt here. The numbers $n(x, y)-n(y, x)$ define a 0 -weighted tournament, which can be considered as a canonical form of weighted tournaments.

Four of the most studied $C 1$ social choice correspondences are: Schwartz's (1972) top cycle (TC), Fishburn's (1977) and Miller's (1980) uncovered set (UC), Dutta's (1988) minimal covering set $(M C)$ and Laffond et al.'s (1993) bipartisan set $(B P)$. Laffond et al. (1995) compare these $C 1$ choice correspondences from a set-theoretical point of view. Given a pair of social choice correspondences, and more precisely the choice sets of these correspondences, they determine which of the following three propositions holds:

- One always contains the other.

- They always intersect but, in some situations, none of them contains the other one.

- In some situations, they have an empty intersection.

Moulin (1986) also discusses some of these relations.

Laffond et al. (1994) introduce the plurality (weighted) bipartisan set $\left(B P_{\mathrm{w}}\right)$. In an article contemporary with ours, Dutta and Laslier (1999) introduce the $C 2$ counterparts of the weighted uncovered set $\left(U C_{\mathrm{w}}\right)$ and the weighted minimal covering set $\left(M C_{\mathrm{w}}\right)$. Our purpose in this article is twofold. First we fill the gap between $C 1$ and $C 2$ correspondences by introducing the weighted top cycle $\left(T C_{\mathrm{w}}\right)$. Our second objective is to perform the set-theoretical comparisons, as in Laffond et al. (1995), between the four $C 1$ correspondences, their four $C 2$ counterparts and three other $C 2$ correspondences frequently encountered in the literature, namely the Kemeny $(\mathrm{Ke})$, the Kramer-Simpson $(S K)$ and the Borda (Bor) rules. Some of these comparisons are borrowed from the existing literature. The other ones, and especially those involving the new $C 2$ concepts, have to be done from scratch. Our findings are summarized in Table 2 at the beginning of Section 3.

There is no obvious way of defining the weighted top cycle $\left(T C_{\mathrm{w}}\right)$ directly in the context of voting situations. Our strategy is the following. As explained above, a voting 
situation induces a tournament and a 0-weighted tournament. Each of these two tournaments induces in turn a two-player zero-sum game for which different solution concepts can be found in the literature. It turns out that the four $C 1$ social choice correspondences mentioned above correspond to four different solution concepts for the game induced by the corresponding tournament. Taking the same solution concepts for the game induced by the corresponding 0-weighted tournament instead of the tournament and working backward from these solution concepts to the solutions for the corresponding 0 -weighted tournament and then to the voting situation yields the corresponding $C 2$ social choice correspondences. This is how the weighted bipartisan set $\left(B P_{\mathrm{w}}\right)$ is defined by Laffond et al. (1994). As Dutta and Laslier (1999), we define the weighted uncovered set $\left(U C_{\mathrm{w}}\right)$ and the weighted minimal covering set $\left(M C_{\mathrm{w}}\right)$ directly in the context of voting situations but in a way that preserves the correspondence with the solution concepts for the plurality game induced by the corresponding 0 -weighted tournament. Finally, the weighted top cycle $\left(T C_{\mathrm{w}}\right)$ is defined as the mixed saddle of the plurality game induced by the corresponding 0 -weighted tournament.

\subsection{Highlights of the results}

Concerning the relations between $T C, U C, M C, B P$ and their weighted counterparts $T C_{\mathrm{w}}, U C_{\mathrm{w}}, M C_{\mathrm{w}}, B P_{\mathrm{w}}$, we show that $U C \subseteq U C_{\mathrm{w}}$ and $M C \subseteq M C_{\mathrm{w}}$. We know from Laffond et al. (1994) that possibly $B P \cap B P_{\mathrm{w}}=\emptyset$. As for the top cycles, we obtain instead that $T C_{\mathrm{w}} \subseteq T C$, i.e. the weighted top cycle is a refinement of the top cycle. It is well known that $B P \subseteq M C \subseteq U C \subseteq T C$. In the weighted case the relations are more intricate. It is easy to see that $B P_{\mathrm{w}} \subseteq U C_{\mathrm{w}}$ and $M C_{\mathrm{w}} \subseteq U C_{\mathrm{w}}$. We show that $B P_{\mathrm{w}} \subseteq T C_{\mathrm{w}}$ and $B P_{\mathrm{w}} \subseteq M C_{\mathrm{w}}$ and also that none of $T C_{\mathrm{w}}$ and $M C_{\mathrm{w}}$ is a superset of the other.

Our main results concerning the Kemeny rule are: $K e$ is a subset of $T C$ and $U C_{\mathrm{w}}$; otherwise this set may have an empty intersection with any of the six remaining sets and notably with $U C$ and $T C_{\mathrm{w}}$. As for the Kramer-Simpson rule, we prove that $S K$ intersects $M C_{\mathrm{w}}$ and therefore $U C_{\mathrm{w}}$. Otherwise, $S K$ may have an empty intersection with any of the six remaining sets and notably with the top cycle. Finally, the set of Borda winners Bor may have an empty intersection with all other sets except with $U C_{\mathrm{w}}$. Actually, Bor is a subset of $U C_{\mathrm{w}}$.

In this article, we restrict ourselves to (asymmetric) tournaments in which no ties are allowed either in the individual encounters or in the split of the victories between contestants. In other words, we assume that the binary relation defining an unweighted tournament is asymmetric and that the numbers $n(x, y)$ defining a $q$-weighted tournament satisfy $n(x, y) \neq n(y, x)$. Furthermore, when discussing the solutions $B P$ and $B P_{\mathrm{w}}$, we restrict ourselves to weighted tournaments for which the numbers $n(x, y)$ are odd integers.

\subsection{Related literature}

A more general class of tournaments consists of weak weighted tournaments. Dutta and Laslier (1999) consider the class of weak 0-weighted tournaments, which they call comparison functions. They introduce the notion of the uncovered set and prove the 
uniqueness of the minimal covering set for these comparison functions. Each comparison function induces a two-player zero-sum symmetric game as in this article. This game has not necessarily a unique Nash equilibrium in mixed strategies. They consider the largest possible support of a Nash equilibrium in mixed strategies, which they call the Essential set of the comparison function. They extend our Propositions $3\left(U C \subseteq U C_{\mathrm{w}}\right), 6$ $\left(M C \subseteq M C_{\mathrm{w}}\right)$, and $7\left(B P_{\mathrm{w}} \subseteq M C_{\mathrm{w}}\right)$ to comparison functions, replacing $B P_{\mathrm{w}}$ with their essential set in the latter. Note, however, that the interpretation of $M C_{\mathrm{w}}$ in terms of saddles does not necessarily extend to all comparison functions.

The 0 -weighted tournaments for which $n(x, y) \in\{-1,0,1\}$ form a special class of comparison functions, called weak tournaments by Peris and Subiza (1999). They also prove, among other results, the uniqueness of the minimal covering set for weak tournaments. Duggan and Le Breton (1997b) look at the different saddles of the weak tournament game. They prove that the essential set is a subset of the (unique) mixed saddle and examine the relations among the different saddles.

\subsection{Contents}

In Section 2, we present the definitions concerning tournaments and voting situations, some non-standard game-theoretical notions, and the 11 solutions for tournaments or weighted tournaments discussed and compared in this article. In Section 3, we proceed to the set-theoretical comparison of these 11 solutions. Finally, we conclude in Section 4 with some lessons that can be drawn from these comparisons.

\section{Definitions}

\subsection{Tournaments, weighted tournaments and situations}

A tournament is a pair $(X, T)$ where $X$ is a finite set and $T$ is an asymmetric and complete binary relation over $X$. Let $\hat{X}^{2} \equiv\left\{(x, y) \in X^{2}: x \neq y\right\}$. Thus, in a tournament, $\forall(x, y) \in \hat{X}^{2}$, we have either $x T y$ or $y T x$, where $x T y$ may be interpreted as: $x$ beats $y$.

Let $q$ be a non-negative real number. A $q$-weighted tournament is a pair $(X, N)$ where $X$ is a finite set and $N$ is a matrix $N \equiv[n(x, y)]_{x, y \in X}$ such that $n(x, y)+n(y, x)=q$ $\forall(x, y) \in X^{2}$ and $n(x, y) \neq n(y, x) \forall(x, y) \in \hat{X}^{2}$. This implies that $n(x, x)=q / 2 \forall x \in X$. A $q$-weighted tournament $(X, N)$ induces the tournament $\left(X, T^{N}\right)$ where $T^{N}$ is defined by: $\forall(x, y) \in \hat{X}^{2}: x T^{N} y$ if and only if $n(x, y)>n(y, x)$. Thus a $q$-weighted tournament may be seen as a tournament with additional information as to how strong $x$ is with respect to $y$.

In this article, we focus on 0 -weighted tournaments. A $q$-weighted tournament $(X, N)$ induces the 0 -weighted tournament $\left(X, M^{N}\right)$ where the elements of $M^{N}$ are defined by

$$
m^{N}(x, y) \equiv n(x, y)-n(y, x)=2 n(x, y)-q .
$$

Conversely, given any $q \geq 0$, a 0 -weighted tournament $(X, M)$ induces the $q$-weighted tournament $\left(X, N^{M}\right)$ where the elements of $N^{M}$ are defined by 


$$
n^{M}(x, y) \equiv \frac{m(x, y)+q}{2}
$$

Clearly, $N^{M^{N}}=N$ and $M^{N^{M}}=M$. For any $q \geq 0$, there is thus a one-to-one correspondence between 0 -weighted tournaments and $q$-weighted tournaments. A tournament $(X, T)$ may be seen as a 0 -weighted tournament $(X, M)$ where, $\forall(x, y) \in \hat{X}^{2}, m(x, y) \in$ $\{-1,1\}$.

Let $\mathscr{T}$ be the set of all tournaments over all finite sets of alternatives. A solution for $\mathscr{T}$ is a multivalued mapping $S_{\mathscr{T}}: \mathscr{T} \rightarrow X$ that assigns a nonempty choice set $S_{\mathscr{T}}(X, T) \subseteq X$ to each tournament $(X, T)$. Similarly, if we let $\mathcal{N}$ be the set of weighted tournaments, a solution for $\mathcal{N}$ is a multivalued mapping $S_{\mathcal{N}}: \mathcal{N} \rightarrow X$ that assigns a nonempty choice set $S_{\mathcal{N}}(X, N) \subseteq X$ to every weighted tournament $(X, N)$. A solution $S_{\mathcal{N}}$ is Condorcet consistent if, for each $q$-weighted tournament $(X, N)$, we have $S_{\mathcal{N}}(X, N)=\{x\}$ whenever $n(x, y)>q / 2$ for all $y \in X, y \neq x$. Alternative $x$ is then called the Condorcet winner.

Consider a solution $S_{\mathcal{M}}$ for the subset $\mathcal{M}$ of 0 -weighted tournaments. Given the one-to-one correspondence between 0 -weighted tournaments and $q$-weighted tournaments for a particular $q$, we can extend the solution $S$ for $\mathcal{M}$ to all other weighted tournaments in $\mathcal{N}$ by $S(X, N) \equiv S\left(X, M^{N}\right)$. The contrary is not possible. A solution for $\mathcal{N}$ may associate two different subsets of $X$ to two different $N$ even if the latter induces the same $M$.

To show that this abstract framework covers voting, we define a (voting) situation as a pair $(X, P)$ where $X$ is a finite set of alternatives and $P$ is a finite list $\left(P_{1}, P_{2}, \ldots, P_{i}, \ldots\right)$ of linear orders on $X$. Their number is noted \#P. These linear orders may be interpreted as the preferences of $\# P$ individuals or voters. Since only preferences matter, we do not introduce voters explicitly in the model. Given a subset $Y \subseteq X, P \mid Y$ represents the restriction of $P$ to $Y$. Given a profile $P$, we define

$$
\begin{aligned}
& \forall(x, y) \in \hat{X}^{2}: n_{P}(x, y) \equiv \#\left\{P_{i} \in P: x P_{i} y\right\} \\
& \forall x \in X: n_{P}(x, x) \equiv \# P / 2, \\
& N_{P} \equiv\left[n_{P}(x, y)\right]_{x, y \in X} .
\end{aligned}
$$

Let $\Omega$ be the set of all possible situations. A social choice correspondence is a multi-valued mapping $\Gamma: \Omega \rightarrow X$ that associates a non-empty choice set $\Gamma(X, P) \subseteq X$ to every situation $(X, P)$. As for tournaments, a social choice correspondence is Condorcet consistent if, for each situation $(X, P)$, we have $\Gamma(X, P)=\{x\}$ whenever $n(x, y)>\# P / 2$ for all $y \in X, y \neq x$. We concentrate on the subclass $\mathscr{D} \subset \Omega$ of situations such that the matrix $N_{P}$ defines a $\# P$-weighted tournament. We write $M_{P}$ for $M^{N_{P}}$ and $T_{P}$ for $T^{N_{P}}$. $\left(X, T_{P}\right)$, $\left(X, N_{P}\right)$ and $\left(X, M_{P}\right)$ are, respectively, the tournament, the $\# P$-weighted tournament, and the 0 -weighted tournament induced by profile $P$ on $X$.

The relation from $\mathscr{D}$ to $\mathscr{T}$ defined by $T \equiv T_{P}$ is onto. Indeed, McGarvey (1953) shows that for any tournament $(X, T)$, there exists a profile $P$ such that $T=T_{P}$. A similar result by Debord (1987) asserts that for any anti-symmetric matrix $M$, there exists a profile $P$ such that $M=M_{P}$ if and only if all the off-diagonal entries of $M$ have the same parity. 
Thus the relation from $\mathscr{D}$ defined by $M \equiv M_{P}$ is not onto the whole set $\mathcal{M}$. Following Barthelemy et al. (1989), we call voting tournaments the 0-weighted tournaments $(X, M)$ such that all the off-diagonal entries of $M$ have the same parity. The relation from $\mathscr{D}$ to $\mathcal{N}$ is more intricate and will not be discussed here. ${ }^{3}$

A social choice correspondence $\Gamma$ is $C 1$ if, for all pairs of situations $(X, P)$ and $\left(X, P^{\prime}\right)$, $T_{P}=T_{P^{\prime}}$ implies $\Gamma(X, P)=\Gamma\left(X, P^{\prime}\right)$. A social choice correspondence $\Gamma$ is $C 2$ if it is not $C 1$ and if for all pairs of situations $(X, P)$ and $\left(X, P^{\prime}\right), M_{P}=M_{P^{\prime}}$ implies $\Gamma(X, P)=$ $\Gamma\left(X, P^{\prime}\right)$. Put differently, a social choice correspondence $\Gamma$ is $C 1$ if there exists a solution $S_{\mathscr{T}}$ for $\mathscr{T}$ such that for all situations $(X, P), \Gamma(X, P)=S_{\mathscr{T}}\left(X, T_{P}\right)$. Similarly, a social choice correspondence $\Gamma$ is $C 2$ if it is not $C 1$ and if there exists a solution $S$ for $\mathcal{M}$ such that for all situations $(X, P), \Gamma(X, P)=S_{\mathcal{M}}\left(X, M_{P}\right)$. As explained in the Introduction, Fishburn (1977), to whom one owes this classification, defines the $C 2$ class with respect to $N_{P}$ instead of $M_{P}$. We justified our use of the above definition in the Introduction.

It has just been seen that the choice sets of $C 1$ and $C 2$ social choice correspondences are actually the choice sets of solutions for, respectively, the tournaments and 0weighted tournaments induced by the voting situations. We shall go one step further and work with zero-sum two-person games that can be defined from these tournaments. ${ }^{4}$ To prepare for this task, we review in the next subsection some non-standard solution concepts for zero-sum two-person games.

\subsection{Solutions for two-player zero-sum games}

This subsection presents solution concepts for two-player zero-sum games. It is based on Shapley (1964) and Duggan and Le Breton (1997a)..

Let $G \equiv\left(X_{1}, X_{2}, u\right)$ be a finite two-player zero-sum game where $X_{1}$ and $X_{2}$ are the sets of pure strategies of players 1 and 2 , respectively, and $u: X_{1} \times X_{2} \rightarrow \mathfrak{R}$ is the payoff function of player 1 . The payoff function of player 2 is $-u$. A game $G$ is symmetric if $X_{1}=X_{2}$ and $u(x, y)+u(y, x)=0$ for all $(x, y) \in X_{1} \times X_{2}$. For any subset $A_{i} \subseteq X_{i}, \Delta\left(A_{i}\right)$ denotes the set of probability distributions over $A_{i}$. Given some vector of probability $p \in \Delta\left(A_{i}\right)$, the support of $p$ is the $\operatorname{set} \operatorname{Supp}(p) \equiv\left\{x \in A_{i}: p(x)>0\right\}$.

Let $A_{2}$ be a subset of strategies for player 2 . The following notions of dominance are classical.

- $x_{1}$ is strictly dominated by $y_{1}$ relative to $A_{2}$ if $u\left(y_{1}, z_{2}\right)>u\left(x_{1}, z_{2}\right)$ for all $z_{2} \in A_{2}$.

- $x_{1}$ is weakly dominated by $y_{1}$ relative to $A_{2}$ if $u\left(y_{1}, z_{2}\right) \geq u\left(x_{1}, z_{2}\right)$ for all $z_{2} \in A_{2}$ with a strict inequality for at least one $z_{2} \in A_{2}$.

- $x_{1}$ is strictly dominated in the mixed sense by $p_{1} \in \Delta\left(X_{1}\right)$ relative to $A_{2}$ if:

$$
\sum_{y_{1} \in X_{1}} p_{1}\left(y_{1}\right) u\left(y_{1}, z_{2}\right)>u\left(x_{1}, z_{2}\right) \text { for all } z_{2} \in A_{2} \text {. }
$$

\footnotetext{
${ }^{3}$ This is the well-known binary stochastic choice problem. See Fishburn (1992) for a description of the state of the art on the latter.

${ }^{4}$ Laffond et al. (1994) give an interpretation of these games as Downsian games in Political Science.

${ }^{5} \mathrm{We}$ restrict ourselves to two-player zero-sum games but these notions can be extended to any finite $n$-player game.
} 
The same three dominance relations are defined similarly for player 2 . The following terminology is borrowed from Shapley (1964) and Duggan and Le Breton (1997a).

Definition 1. A Generalized Saddle Point (GSP) for a game $G$ is a product $A_{1} \times A_{2} \subseteq$ $X_{1} \times X_{2}$ such that:

$$
\begin{aligned}
& \forall x_{1} \notin A_{1}: \exists y_{1} \in A_{1} \text { such that } x_{1} \text { is strictly dominated by } y_{1} \text { relative to } A_{2} \text {, } \\
& \forall x_{2} \notin A_{2}: \exists y_{2} \in A_{2} \text { such that } x_{2} \text { is strictly dominated by } y_{2} \text { relative to } A_{1} .
\end{aligned}
$$

A GSP that does not contain other GSP is a Saddle.

Definition 2. A Weak Generalized Saddle Point (WGSP) for a game $G$ is a product $A_{1} \times A_{2} \subseteq X_{1} \times X_{2}$ such that:

$$
\begin{aligned}
& \forall x_{1} \notin A_{1}: \exists y_{1} \in A_{1} \text { such that } x_{1} \text { is weakly dominated by } y_{1} \text { relative to } A_{2} \text {, } \\
& \forall x_{2} \notin A_{2}: \exists y_{2} \in A_{2} \text { such that } x_{2} \text { is weakly dominated by } y_{2} \text { relative to } A_{1} \text {. }
\end{aligned}
$$

A WGSP that does not contain other WGSP is a Weak Saddle.

Definition 3. A Mixed Generalized Saddle Point (MGSP) for a game $G$ is a product $A_{1} \times A_{2} \subseteq X_{1} \times X_{2}$ such that:

$$
\forall x_{1} \notin A_{1}: \exists p_{1} \in \Delta\left(A_{1}\right)
$$

such that $x_{1}$ is strictly dominated in the mixed sense by $p_{1}$ relative to $A_{2}$,

$$
\forall x_{2} \notin A_{2}: \exists p_{2} \in \Delta\left(A_{2}\right)
$$

such that $x_{2}$ is strictly dominated in the mixed sense by $p_{2}$ relative to $A_{1}$.

A MGSP that does not contain other MGSP is a Mixed Saddle.

Since $X_{1}$ and $X_{2}$ are assumed to be finite, saddles, weak saddles, and mixed saddles exist. Shapley (1964) proves that there exists a unique saddle and Duggan and Le Breton (1997a) prove that there is a unique mixed saddle. A two-player zero-sum game may have several weak saddles. However, Duggan and Le Breton (1996) prove that if $G$ is symmetric and $u(x, y) \neq 0$ for all $x \neq y$, then there is a unique weak saddle. Moreover, if $G$ is symmetric, the saddle, the weak saddle (if unique) and the mixed saddle are symmetric, i.e. of the form $A \times A$. In this case, we shall refer to $A$ as the saddle, weak saddle or mixed saddle.

Definition 4. An equilibrium in mixed strategies for a game $G$ is a pair of probability distributions $p_{1} \in \Delta\left(X_{1}\right)$ and $p_{2} \in \Delta\left(X_{2}\right)$ that satisfy the following inequalities: 


$$
\begin{aligned}
& \sum_{x_{1} \in X_{1} x_{2} \in X_{2}} p_{1}\left(x_{1}\right) p_{2}\left(x_{2}\right) u\left(x_{1}, x_{2}\right) \geq \sum_{x_{1} \in X_{1} x_{2} \in X_{2}} \sum_{1}\left(x_{1}\right) p_{2}\left(x_{2}\right) u\left(x_{1}, x_{2}\right), \forall q_{1} \in \Delta\left(X_{1}\right), \\
& \sum_{x_{1} \in X_{1} x_{2} \in X_{2}} \sum_{1} p_{1}\left(x_{1}\right) p_{2}\left(x_{2}\right) u\left(x_{1}, x_{2}\right) \leq \sum_{x_{1} \in X_{1} x_{2} \in X_{2}} \sum_{1} p_{1}\left(x_{1}\right) q_{2}\left(x_{2}\right) u\left(x_{1}, x_{2}\right), \forall q_{2} \in \Delta\left(X_{2}\right) .
\end{aligned}
$$

The following result, known as the Minmax Theorem, provides a characterization of equilibria in mixed strategies. See Owen (1982) for a proof.

Lemma 1. Let $\vartheta \equiv \min _{p_{2} \in \Delta\left(X_{2}\right)} \max _{p_{1} \in \Delta\left(X_{1}\right)} \Sigma_{x_{1} \in X_{1}} \Sigma_{x_{2} \in X_{2}} p_{1}\left(x_{1}\right) p_{2}\left(x_{2}\right) u\left(x_{1}, x_{2}\right)$. A pair $\left(p_{1}, p_{2}\right) \in \Delta\left(X_{1}\right) \times \Delta\left(X_{2}\right)$ is an equilibrium in mixed strategies if and only if:

$$
\begin{aligned}
& \sum_{x \in X_{1}} p_{1}(x) u(x, y) \geq \vartheta, \forall y \in X_{2}, \\
& \sum_{y \in X_{2}} p_{2}(y) u(x, y) \leq \vartheta, \quad \forall x \in X_{1} .
\end{aligned}
$$

The scalar $\vartheta$ in the above lemma is the value of the game. If $G$ is symmetric then $\vartheta=0$. Laffond et al. (1997) prove that if $G$ is symmetric and $u(x, y)$ is an odd integer for all $x \neq y$, then $G$ has a unique Nash equilibrium in mixed strategies.

\subsection{Tournament games and C1 choice correspondences}

A tournament $(X, T)$ induces a symmetric two-player zero-sum game $\left(X, X, u_{T}\right)$ where:

$$
u_{T}(x, y) \equiv \begin{cases}1, & \text { if } x T y \\ -1, & \text { if } y T x \\ 0, & \text { if } x=y\end{cases}
$$

We call this game the tournament game induced by $(X, T)$. The relation between tournaments and tournament games is clearly one-to-one. Since a tournament game is symmetric and $u_{T}(x, y)$ is an odd integer for all $x \neq y$, we know that it has a unique saddle, a unique weak saddle, a unique mixed saddle, and a unique equilibrium in mixed strategies. Duggan and Le Breton (1997b) point out that if $T$ has no Condorcet winner, then the saddle of the tournament game consists of the whole set $X$, which is not very discriminating. We shall therefore focus on the other two saddles.

Next, we introduce three $C 1$ solution concepts for tournaments. As we shall see, each has a connection with a solution of the corresponding tournament game. The first of these solutions is Schwartz's (1972) top cycle.

Definition 5. The top cycle $T C(X, T)$ of a tournament $(X, T)$ is the set of all outcomes that beat directly or indirectly any other outcome in $X$ :

$$
T C(X, T) \equiv\left\{\begin{array}{l}
a \in X: \forall b \in X /\{a\}, \exists a_{1}, a_{2}, \ldots, a_{k} \in X \\
\text { such that } a=a_{1} T a_{2} T \ldots T a_{k}=b
\end{array}\right\} .
$$


Duggan and Le Breton (1997b) prove that the mixed saddle of the tournament game induced by $(X, T)$ is the top cycle of $(X, T)$.

Fishburn (1977) and Miller (1980) propose another tournament solution called the uncovered set. They first define the covering relation $C$ relative to a subset $Y \subseteq X$ as: $\forall x, y \in X, x C(T) y$ relative to $Y \subseteq X$ if $x T y$ and if $y T w \Rightarrow x T w, \forall w \in Y$.

Definition 6. The uncovered set $U C(X, T)$ of a tournament $(X, T)$ is the set of maximal elements of the covering relation $C(T)$ relative to $X$ :

$$
U C(X, T) \equiv\{a \in X: \exists b \in X: b C(T) a \text { relative to } X\} \text {. }
$$

The covering relation is also the transposition, to tournaments, of the weak dominance relation of the corresponding tournament game. Thus, the uncovered set of a tournament $(X, T)$ is also the set of weakly undominated strategies (relative to $X)$ in the corresponding tournament game $\left(X, X, u_{T}\right)$.

We can refine this solution concept by iteration. Let $U C^{0}(X, T) \equiv X$ and, for all integers $t \geqq 1$, let:

$$
U C^{t}(X, T) \equiv U C\left(U C^{t-1}(X, T), P \mid U C^{t-1}(X, T)\right) .
$$

Let $k$ be the smallest integer such that $U C^{k+1}(X, T)=U C^{k}(X, T)$ and let $\overline{U C}(X, T) \equiv$ $U C^{k}(X, T)$. Clearly, $\overline{U C}(X, T) \subseteq U C(X, T)$ for any tournament.

Dutta (1988) proposes another solution concept, the minimal covering set, also based on the covering relation $C(T)$. First, he defines a covering set of a tournament $(X, T)$ as a subset $A \subseteq X$ satisfying:

$$
U C(X, T \mid A)=A \text { and } \forall b \notin A: b \notin U C(X, T \mid A \cup\{b\}) .
$$

We can reformulate these two conditions as stability conditions. The first is an internal stability condition:

$$
\forall x, y \in A, \text { not } x C(T) y \text { relative to } A \text { and not } y C(T) x \text { relative to } A,
$$

i.e. no option in $A$ is covered relative to $A$. The second is an external stability condition:

$\forall b \notin A, \exists y \in A: y C(T) b$ relative to $A \cup\{b\}$.

It is easy to see that $\overline{U C}(X, T)$ is a covering set. We also define a weak covering set of a tournament $(X, T)$ as a subset $A \subseteq X$ such that $\forall x \notin A, \exists y \in A: y C(T) x$ on $A \cup\{x\}$. A weak covering set satisfies external stability but not necessarily internal stability. Clearly, a weak covering set is a WGSP of the corresponding tournament game.

Next, Dutta defines a minimal covering set of a tournament $(X, T)$ as a covering set of $(X, T)$ that does not contain other covering sets of $(X, T)$. Since $\overline{U C}(X, T)$ is a covering set and since $X$ is finite, minimal covering sets do exist. Dutta proves that there is in fact a unique minimal covering set that will be denoted $M C(X, T)$. Thus, we have the following definition. 
Definition 7. The minimal covering set $M C(X, T)$ of a tournament $(X, T)$ is the unique covering set of $(X, T)$ that does not contain other covering sets of $(X, T)$.

Duggan and Le Breton (1996) show that $M C(X, T)$ is the weak saddle of the tournament game induced by $(X, T)$. The last $C 1$ solution to be introduced in this subsection is due to Laffond et al. (1993). It is transposed from the solution of the corresponding tournament game.

Definition 8. The Bipartisan set of a tournament $(X, T)$ is the support $B P(X, T)$ of the unique Nash equilibrium in mixed strategies of the tournament game $\left(X, X, u_{T}\right)$ induced by this tournament.

\subsection{Plurality games and C2 choice correspondences}

As for tournaments, a 0 -weighted tournament $(X, M)$ induces a symmetric two-player zero-sum game $\left(X, X, u_{M}\right)$, where

$$
u_{M}(x, y) \equiv m(x, y) \text { for all } x, y \in X
$$

We call this game the plurality game induced by $(X, M)$. The relation between 0 -weighted tournaments and plurality games is clearly one-to-one. The plurality game has a unique saddle and, in contrast to the tournament game, the saddle may be a proper subset of $X$. We shall not discuss this set in this article, to keep a symmetric treatment of the two kinds of games.

The result concerning the mixed saddle of the tournament game suggests the following definition, for which there is no obvious intuition.

Definition 9. The weighted top cycle $T C_{\mathrm{w}}(X, M)$ of a 0 -weighted tournament $(X, M)$ is the mixed saddle of the plurality game induced by $(X, M)$.

We now transpose the covering relation of the previous subsection to 0 -weighted tournaments. We define the weighted covering relation $C_{\mathrm{w}}(M)$ relative to a subset $Y \subseteq X$ as follows:

$$
\forall x, y \in X, x C_{\mathrm{w}}(M) y \text { relative to } Y \subseteq X \text { if } m(x, y)>0 \text { and if } m(x, z) \geq m(y, z), \forall z \in Y \text {. }
$$

Definition 10. The weighted uncovered set $U C_{\mathrm{w}}(X, M)$ of a 0 -weighted tournament $(X, M)$ is the set of maximal elements of the weighted covering relation $C_{\mathrm{w}}(M)$ relative to $X:$

$$
U C_{\mathrm{w}}(X, M) \equiv\left\{a \in X: \exists b \in X: b C_{\mathrm{w}}(M) a \text { relative to } X\right\}
$$

The weighted covering relation is also the transposition, to 0-weighted tournaments, of the weak dominance relation of the corresponding plurality game. Thus, the weighted uncovered set of a 0 -weighted tournament $(X, M)$ could equivalently be defined as the set 
of weakly undominated strategies (relative to $X$ ) in the corresponding plurality game $\left(X, X, u_{M}\right)$.

We can also define the iterates of this set, namely $U C_{\mathrm{w}}^{1}(X, M), U C_{\mathrm{w}}^{2}(X, M)$, etc., as we did for $U C(X, T)$. The limit $\overline{U C}_{\mathrm{w}}(X, M)$ of this series is the set of strategies that remain after iterative elimination of weakly dominated strategies.

Next, we use the weighted covering relation $C_{\mathrm{w}}$ to introduce the weighted equivalent of the minimal covering set. A weighted covering set of a 0 -weighted tournament $(X, M)$ is a subset $A \subseteq X$ satisfying:

$$
U C_{\mathrm{w}}(X, M \mid A)=A \text { and } \forall x \notin A: x \notin U C_{\mathrm{w}}(X, M \mid A \cup\{x\}) .
$$

Note that $\overline{U C}_{\mathrm{w}}(X, M)$ is a weighted covering set. We also define a weak weighted covering set of a 0 -weighted tournament $(X, M)$ as a subset $A \subseteq X$ such that $\forall x \notin A$, $\exists y \in A: y C_{\mathrm{w}}(M) x$ relative to $A \cup\{x\}$. Clearly, a weak weighted covering set of $(X, M)$ is a WGSP of the plurality game induced by $(X, M)$. The following simple lemma establishes the relation between weak weighted covering sets and weak covering sets.

Lemma 2. If $A$ is a weak weighted covering set of a 0 -weighted tournament $(X, M)$, then $A$ is also a weak covering set of the tournament $\left(X, T^{M}\right)$.

Proof. Let $A$ be a weak weighted covering set of a 0 -weighted tournament and take any $x \notin A$. By definition, $\exists y \in A: m(y, z) \geq m(x, z), \forall z \in A \cup\{x\}$. Thus, $x T^{M} z \Rightarrow m(x, z)>$ $0 \Rightarrow m(y, z)>0 \Rightarrow y T^{M} z$. This means that $y C\left(T^{M}\right) x$ relative to $A \cup\{x\}$.

A minimal weighted covering set of a 0 -weighted tournament $(X, M)$ is a weighted covering set that does not contain other covering sets of $(X, M)$. Since $\overline{U C}_{\mathrm{w}}(X, M)$ is a weighted covering set and since $X$ is finite, minimal weighted covering sets do exist. In order to justify the next definition, we need to show the following result.

Lemma 3. A minimal (with respect to inclusion) weak covering set of a tournament is also a minimal covering set of the same tournament. Similarly, a minimal weak weighted covering set of a 0 -weighted tournament is also a minimal weighted covering set of the same 0-weighted tournament.

Proof. Let $A$ be a minimal weak covering set of a tournament. By definition, $A$ satisfies the external stability condition. We claim that it also satisfies the internal stability condition. Suppose not, i.e. $\exists x, y \in A: x C y$ relative to $A$. Since $C$ is transitive, $y$ can be removed from $A$ without sacrificing external stability, contradicting the assumption that $A$ is a minimal weak covering set. Thus $A$ is a minimal covering set. The same arguments apply to minimal weighted weak covering sets.

Since the plurality game induced by a 0 -weighted tournament $(X, M)$ has a unique weak saddle, it has a unique minimal weak weighted covering set, which must also be the unique minimal weighted covering set of $(X, M)$ by Lemma 3 . Thus, we may state the following definition. 
Definition 11. The minimal weighted covering set $M C_{\mathrm{w}}(X, M)$ of a 0 -weighted tournament $(X, M)$ is the unique weighted covering set of $(X, M)$ that does not contain other covering sets of $(X, M)$.

Finally, under the additional restriction that the elements $m(x, y)$ of $M$ are odd integer for all $x \neq y$, the plurality game has a unique equilibrium in mixed strategies. The support of this equilibrium is another solution for 0 -weighted tournaments.

Definition 12. The Weighted Bipartisan Set $B P_{\mathrm{w}}(X, M)$ of a 0 -weighted tournament $(X, M)$ is the support of the unique equilibrium in mixed strategies of the corresponding plurality game.

We list the different solution sets for tournament and plurality games in Table 1. Recall that $X$ is a saddle for a tournament only if there is no Condorcet winner.

\subsection{Three other $C 2$ choice correspondences}

We now present three $C 2$ social choice correspondences that have been widely discussed in the literature and that are very often used. Their transposition as solutions for a weighted tournament will be immediate.

The first concept is due to Kemeny (1959). Given a situation $(X, P)$, this correspondence first chooses the linear orders that are as close as possible to $P$ in a sense to be made precise below and then takes the top elements of these orders as the choice set. Given a set $X$ and two linear orders $O$ and $O^{\prime}$ on $X$, let

$$
\delta\left(O, O^{\prime}\right) \equiv \#\left\{(x, y) \in X^{2}, x \neq y, x O y \text { and } y O^{\prime} x\right\} .
$$

In words, $\delta\left(O, O^{\prime}\right)$ is the number of inversions in the two orders $O$ and $O^{\prime}$. Clearly, $\delta$ is a distance over the set of orders on $X$. Kemeny then defines a 'distance' between a linear order $O$ and a profile $P$ as:

$$
d(O, P) \equiv \sum_{P_{i} \in P} \delta\left(O, P_{i}\right)
$$

Table 1

Solution concepts for tournament and plurality games

\begin{tabular}{lll}
\hline Game & Tournament & Plurality \\
\hline Saddle & $X$ & \\
Weak saddle & $M C$ & $M C_{\mathrm{w}}$ \\
Mixed saddle & $T C$ & $T C_{\mathrm{w}}$ \\
Weakly undominated strategies & $U C$ & $U C_{\mathrm{w}}$ \\
Support of the unique equilibrium & & \\
$\quad$ in mixed strategies & $B P$ & $B P_{\mathrm{w}}$ \\
\hline
\end{tabular}

${ }^{6}$ This definition was first proposed by Laffond et al. (1994). 
Definition 13. A Kemeny order for a situation $(X, P)$ is a linear order $O^{*} \in$ $\arg \min _{O \in L} d(O, P)$. The Kemeny set $K e(X, P)$ of a situation $(X, P)$ is the set of top elements of the Kemeny orders for the situation.

The following simple and well-known lemma gives a useful characterization of the Kemeny choice correspondence.

Lemma 4. $O *$ is a Kemeny order for a situation $(X, P)$ if and only if

$$
O^{*} \in \arg \max _{O \in L} \sum_{x \in X y \in X} \sum_{\substack{x O y \\ x}} n_{P}(x, y) .
$$

It follows from this lemma that the Kemeny social choice correspondence is $C 2$. This lemma inspires the following definition for weighted tournaments.

Definition 14. A Kemeny order for a $q$-weighted tournament $(X, N)$ is a linear order

$$
O * \in \arg \max _{O \in L} \sum_{x \in X y \in X} \sum_{x O y} n(x, y) .
$$

The Kemeny set $\overline{K e}(X, N)$ of a $q$-weighted tournament $(X, N)$ is the set of the top elements of the Kemeny orders for the weighted tournament.

Since

$$
\arg \max _{O \in L} \sum_{x \in X y \in X} \sum_{x O y} n_{P}(x, y)=\arg \max _{O \in L} \sum_{x \in X y \in X} \sum_{\substack{x \\ x O y}} m_{P}(x, y),
$$

the following relation holds for all situations:

$$
K e(X, P)=\overline{K e}\left(X, N_{P}\right)=\overline{K e}\left(X, M_{P}\right) .
$$

Since there is no risk of confusion, we shall write $\operatorname{Ke}(X, N)$ instead of $\overline{\operatorname{Ke}}(X, N)$ in the remainder of the article.

The Kemeny rule has been axiomatized by Young and Levenglick (1978). Young (1988) also provides a very nice foundation for this procedure. Suppose that there is a true ordering $O$ of the alternatives in $X$ and that in any pairwise comparison, each voter chooses the better candidate with some fixed probability $p$ (the competence parameter), where $\frac{1}{2}<p<1$ and $p$ is the same for all voters. Assume also that every voter's judgment on every pair of candidates is independent of his judgment on every other pair and that judgments are independent from one voter to another. Under these assumptions, it can be shown that $O^{*}$ is a Kemeny order if and only if it maximizes the likelihood of being the true order $O$ given the pattern of pairwise votes in the profile $P$. This is precisely the statistical framework used by Condorcet to justify his criterion.

Slater (1961) proposes a choice correspondence for tournaments. A Slater order for a tournament $(X, T)$ is an order $O^{*} \in \arg \min _{O \in L} \delta(O, T)$. The Slater set $\operatorname{Sl}(X, T)$ for a tournament $(X, T)$ is the set of top elements of the Slater orders for the tournament. Given a 0 -weighted tournament $(X, M)$ such that all the off-diagonal entries of $M$ are 
either -1 or 1 , the Kemeny set for $(X, M)$ and the Slater set for the tournament $\left(X, T^{M}\right)$ coincide. Thus, the Slater rule may be seen as the $C 1$ counterpart of the Kemeny rule.

Simpson (1969) and Kramer (1977) propose to take the set of outcomes whose maximal opposition is the weakest as the solution of a situation. Kramer (1977) shows that this set is an attraction point of a sequential electoral competition between two parties when platforms belong to some Euclidean space.

Definition 15. The Simpson-Kramer set or the minmax set $S K(X, P)$ of a situation $(X, P)$ is:

$$
S K(X, P) \equiv \underset{x \in X}{\arg \min } \max _{y \in X \backslash\{x\}} n_{p}(x, y)
$$

This social choice correspondence is also $C 2$. We can thus transpose its definition to weighted tournaments as we did with the Kemeny rule and a relation similar to (1) holds for the minmax sets.

The last choice correspondence to be introduced is the Borda rule, a well-known scoring method. Given a situation $(X, P)$, we first define: $\forall P_{i} \in P, \forall x \in X, R\left(x, P_{i}\right) \equiv$ $\#\left\{y \in X: x P_{i} y\right\}$. The Borda score of an element $x \in X$ is then defined as $B(x, P) \equiv$ $\Sigma_{P_{i} \in P} R\left(x, P_{i}\right)$.

Definition 16. A Borda winner for a situation $(X, P)$ is any $x^{*} \in \arg \max _{x \in X} B(x, P)$. The Borda set $\operatorname{Bor}(X, P)$ of a situation $(X, P)$ is the set of Borda winners for this situation.

The following lemma is well known.

Lemma 5. For any situation $(X, P), \operatorname{Bor}(X, P)=\arg \max _{x \in X} \sum_{y \in X /\{x\}} n_{P}(x, y)$.

Thus, the Borda correspondence is $C 2$. $^{7}$ As for the Kemeny rule and the KramerSimpson rule, the definition of the Borda rule can be transposed to weighted tournaments and a relation similar to (1) holds for the Borda sets.

Many justifications have been given for the Borda rule. One of them emerges from the statistical framework of Condorcet. Suppose that instead of searching for the 'true' ranking of the candidates, we focus on the determination of which candidates are most likely to be the best. Then, as pointed out by Condorcet (1785) and by Young (1988), if the competence parameter $p$ is sufficiently close to $\frac{1}{2}$, the Borda winners turn out to be the most likely best candidates. However, if $p$ is sufficiently close to 1 , the answer is the Kramer-Simpson set.

Condorcet (1785) showed that the Borda choice correspondence does not always select the Condorcet winner when it exists. The Borda rule is not the only choice correspondence studied here not to be Condorcet consistent. The following example shows that $U C_{\mathrm{w}}$ is not Condorcet consistent. Consider the 0 -weighted tournament defined by the following table:

\footnotetext{
${ }^{7}$ Lemma 5 shows that, in some sense, the Borda set is the $C 2$ conterpart of the Copeland (1951) set for tournaments.
} 


\begin{tabular}{lllll}
\hline & $a$ & $b$ & $c$ & $d$ \\
\hline$a$ & 0 & 1 & 1 & 1 \\
$b$ & -1 & 0 & 5 & -5 \\
$c$ & -1 & -5 & 0 & 5 \\
$d$ & -1 & 5 & -5 & 0 \\
\hline
\end{tabular}

It is easy to see that $\{a\}$ is the Condorcet winner and that $U C_{\mathrm{w}}(X, M)=\{a, b, c, d\}$. All other solutions defined in this section are Condorcet consistent.

\section{Set-theoretical comparison of solution concepts}

In this section, we examine the relationships between the 11 solutions for 0 -weighted tournaments introduced in the previous section. Table 2 summarizes the results of these set-theoretical comparisons.

- A $\subseteq$ in a cell indicates that the solution set of the corresponding row is contained in the solution set of the corresponding column. For the $B P_{\mathrm{w}}$ row, the inclusion is true for any voting tournament with odd $m(x, y) \forall x \neq y$. For all other cells, it is true for any 0 -weighted tournament $(X, M)$.

- A $\cap$ means that for any 0-weighted tournament $(X, M)$, the solution set of the corresponding row intersects the solution set of the corresponding column but that there exists a voting tournament $(X, M)$ such that none of the solution sets of the row or the column is a subset of the other.

- A $\emptyset$ means that there exists a voting tournament $(X, M)$ such that the intersection of the solution sets of the row and the column is empty.

We recall that voting tournaments are 0-weighted tournaments $(X, M)$ such that all the off-diagonal entries of $M$ have the same parity. By Debord's theorem, there always exists a voting situation underlying a voting tournament. Thus, results such as $S \subset S^{\prime}$,

Table 2

Comparison of solution concepts

\begin{tabular}{|c|c|c|c|c|c|c|c|c|c|c|}
\hline & $T C$ & $T C_{w}$ & $U C_{w}$ & $M C_{w}$ & $B P_{w}$ & $U C$ & $M C$ & $K e$ & $S K$ & $B P$ \\
\hline$T C_{w}$ & $\subseteq$ & & & & & & & & & \\
\hline$U C_{w}$ & $\bar{\cap}$ & $\cap$ & & & & & & & & \\
\hline$M C_{w}$ & $\cap$ & $\cap$ & $\subseteq$ & & & & & & & \\
\hline$B P_{w}$ & $\subseteq$ & $\subseteq$ & $\subseteq$ & $\subseteq$ & & & & & & \\
\hline$U C$ & $\subseteq$ & $\emptyset$ & $\subseteq$ & $\bar{\cap}$ & $\emptyset$ & & & & & \\
\hline$M C$ & $\subseteq$ & $\emptyset$ & $\subseteq$ & $\subseteq$ & $\emptyset$ & $\subseteq$ & & & & \\
\hline$K e$ & $\subseteq$ & $\emptyset$ & $\subseteq$ & $\emptyset$ & $\emptyset$ & $\emptyset$ & $\emptyset$ & & & \\
\hline$S K$ & $\emptyset$ & $\emptyset$ & $\cap$ & $\cap$ & $\emptyset$ & $\emptyset$ & $\emptyset$ & $\emptyset$ & & \\
\hline$B P$ & $\subseteq$ & $\emptyset$ & $\subseteq$ & $\subseteq$ & $\emptyset$ & $\subseteq$ & $\subseteq$ & $\emptyset$ & $\emptyset$ & \\
\hline Bor & $\bar{\emptyset}$ & $\emptyset$ & $\subseteq$ & $\bar{\emptyset}$ & $\emptyset$ & $\emptyset$ & $\bar{\emptyset}$ & $\emptyset$ & $\emptyset$ & $\emptyset$ \\
\hline
\end{tabular}


$S \cap S=\emptyset$, and $S \nsubseteq S$ for some voting tournament, where $S$ and $S^{\prime}$ are two solution sets, can be transposed to social choice correspondences.

Remark 1. The chain of inclusions $B P \subseteq M C \subseteq U C \subseteq T C$ is well known from the literature on tournament solutions. Fishburn (1977) shows that Ke $\subseteq$ TC and Laffond et al. (1994) prove that we may have $B P \cap B P_{w}=\emptyset$. The possible empty intersection of Bor with $T C, T C_{w}, M C_{w}, B P_{w}, U C, M C, K e, S K$, and BP follows from the fact that these nine solutions are Condorcet consistent and that a Condorcet winner may fail to be a Borda winner. 9

The remainder of this section deals with the other entries in the table. Many of the inclusion results may be deduced from a glance at Table 2. These deductions are left to the reader. The two examples that follow will be used to establish some of the results.

Example 1. Consider the following voting tournament $(X, M)$ :

\begin{tabular}{llllll}
\hline & $a$ & $b$ & $c$ & $d$ & $e$ \\
\hline$a$ & 0 & 1 & -5 & -3 & 1 \\
$b$ & -1 & 0 & 3 & -3 & 1 \\
$c$ & 5 & -3 & 0 & 3 & 1 \\
$d$ & 3 & 3 & -3 & 0 & 1 \\
$e$ & -1 & -1 & -1 & -1 & 0 \\
\hline
\end{tabular}

One can check that $T C\left(X, T^{M}\right)=\{a, b, c, d\}, T C_{\mathrm{w}}(X, M)=\{b, c, d\}, U C_{\mathrm{w}}(X, M)=\{b, c, d, e\}$, $\operatorname{Ke}(X, M)=\operatorname{Bor}(X, M)=\{c\}$, and $S K(X, M)=\{e\}$.

Example 2. Consider the following voting tournament $(X, M)$ :

\begin{tabular}{lllllll}
\hline & $a$ & $b$ & $c$ & $d$ & $e$ & $f$ \\
\hline$a$ & 0 & 3 & -5 & -3 & 1 & -1 \\
$b$ & -3 & 0 & 3 & -3 & 1 & 1 \\
$c$ & 5 & -3 & 0 & 3 & 1 & -3 \\
$d$ & 3 & 3 & -3 & 0 & 1 & 3 \\
$e$ & -1 & -1 & -1 & -1 & 0 & -1 \\
$f$ & 1 & -1 & 3 & -3 & 1 & 0 \\
\hline
\end{tabular}

One can check that $T C\left(X, T^{M}\right)=T C_{\mathrm{w}}(X, M)=\{a, b, c, d, f\}, \quad U C\left(X, T^{M}\right)=\{b, c, d, f\}$, $U C_{\mathrm{w}}(X, M)=\{b, c, d, e, f\}, \quad M C\left(X, T^{M}\right)=B P\left(X, T^{M}\right)=B P_{\mathrm{w}}(X, M)=\{b, c, d\}, \quad M C_{\mathrm{w}}(X, M)=$ $\{b, c, d, e\}, \operatorname{Ke}(X, M)=\operatorname{Bor}(X, M)=\{d\}$, and $S K(X, M)=\{e\}$.

\footnotetext{
${ }^{8} A \subseteq B$ signifies that $A$ is a subset of $B$ with the possibility that $A=B$ and $\subset$ indicates a strict inclusion.

${ }^{9}$ See the proof of Theorem 4 in Le Breton and Truchon (1997) for a general example.
} 


\subsection{The weighted top cycle $\left(T C_{\mathrm{w}}\right)$}

Proposition 1. $T C_{w}(X, M) \subseteq T C\left(X, T^{M}\right)$ for any 0-weighted tournament $(X, M)$.

Proof. Consider the plurality game induced by $(X, M)$ restricted to $T C\left(X, T^{M}\right)$. Since the value of this game is zero, we deduce from the minmax theorem that there exists a $p \in \Delta\left(T C\left(X, T^{M}\right)\right)$ such that $\Sigma_{x \in T C} p(x) m(x, y) \geq 0$ for all $y \in T C\left(X, T^{M}\right)$. Next take a $z \notin T C\left(X, T^{M}\right)$. From the definition of $T C\left(X, T^{M}\right)$, we have $m(z, y)<0$ for all $y \in$ $T C\left(X, T^{M}\right)$. Combining the two sets of inequalities, we deduce that $z$ is strictly dominated in the mixed sense relative to $T C\left(X, T^{M}\right)$. Therefore, $T C\left(X, T^{M}\right)$ is a MGSP and since $T C_{\mathrm{w}}(X, M)$ is the unique mixed saddle, then $T C_{\mathrm{w}}(X, M) \subseteq T C\left(X, T^{M}\right)$.

Remark 2. Example 1 shows that one can have $T C_{w}(X, M) \subset T C\left(X, T^{M}\right)$.

Proposition 2. There exists a voting tournament $(X, M)$ such that $\operatorname{TC}_{w}(X, M) \cap$ $B P\left(X, T^{M}\right)=T C_{w}(X, M) \cap M C\left(X, T^{M}\right)=T C_{w}(X, M) \cap U C\left(X, T^{M}\right)=\emptyset$.

Proof. Consider the following voting tournament $(X, M)$, which Laffond et al. (1994) use to prove that we may have $B P \cap B P_{\mathrm{w}}=\emptyset$ :

\begin{tabular}{lllllll}
\hline & $a$ & $b$ & $c$ & $x$ & $y$ & $z$ \\
\hline$a$ & 0 & 1 & -1 & 1 & -9 & 1 \\
$b$ & -1 & 0 & 1 & 1 & 1 & -9 \\
$c$ & 1 & -1 & 0 & -9 & 1 & 1 \\
$x$ & -1 & -1 & 9 & 0 & 5 & -5 \\
$y$ & 9 & -1 & -1 & -5 & 0 & 5 \\
$z$ & -1 & 9 & -1 & 5 & -5 & 0 \\
\hline
\end{tabular}

The reader can check that $B P\left(X, T^{M}\right)=M C\left(X, T^{M}\right)=U C\left(X, T^{M}\right)=\{a, b, c\} \quad$ and $B P_{\mathrm{w}}(X, M)=T C_{\mathrm{w}}(X, M)=\{x, y, z\}^{10}$

\subsection{The weighted uncovered set $\left(U C_{\mathrm{w}}\right)$}

It has been shown that the top cycle is a superset of the weighted top cycle. This order of inclusion is reversed for the uncovered set.

Proposition 3. $U C\left(X, T^{M}\right) \subseteq U C_{w}(X, M)$ for any 0-weighted tournament $(X, M)$.

Proof. The proof is straightforward and left to the reader.

\footnotetext{
${ }^{10} a$ is strictly dominated in the mixed sense relative to $\{x, y, z\}$ by $p(x)=0, p(y)=3 / 10, p(z)=7 / 10$.

$b$ is strictly dominated in the mixed sense relative to $\{x, y, z\}$ by $p(x)=7 / 10, p(y)=0, p(z)=3 / 10$.

$c$ is strictly dominated in the mixed sense relative to $\{x, y, z\}$ by $p(x)=3 / 10, p(y)=7 / 10, p(z)=0$.
} 
Remark 3. Example 2 shows that there exists a voting tournament $(X, M)$ such that $U C\left(X, T^{M}\right) \subset U C_{w}(X, M)$.

Proposition 4. (a) $U C_{w}(X, M) \cap T C\left(X, T^{M}\right) \neq \emptyset$ for any 0 -weighted tournament $(X, M)$.

(b) There exists a voting tournament $(X, M)$ such that $U C_{w}(X, M) \nsubseteq T C\left(X, T^{M}\right)$ and $T C\left(X, T^{M}\right) \nsubseteq U C_{w}(X, M)$.

Proof. (a) $K e(X, M)$ is a subset of both $U C_{\mathrm{w}}(X, M)$ and $T C\left(X, T^{M}\right)$ by, respectively, Proposition 10 below and Remark 1 .

(b) This follows from Example 2.

Proposition 5. $\operatorname{Bor}(X, M) \subseteq U C_{w}(X, M)$ for any 0-weighted tournament $(X, M)$.

Proof. If $U C_{\mathrm{w}}(X, M)=X$, there is nothing to prove. Suppose $U C_{\mathrm{w}}(X, M) \neq X$ and take any $y \notin U C_{\mathrm{w}}(X, M)$. By transitivity of $C_{\mathrm{w}}$, there must exist an $x \in U C_{\mathrm{w}}(X, M)$ such that $x C_{\mathrm{w}} y$ relative to $X$, i.e. such that $m(x, y)>0$ and $m(x, z)-m(y, z) \geq 0 \forall z \neq x, y$. This implies $\Sigma_{z \in X} m(x, z)>\Sigma_{z \in X} m(y, z)$ and thus $y$ is not a Borda winner.

Remark 4. Examples 1 and 2 both show that there exists a voting tournament $(X, M)$ such that $\operatorname{Bor}(X, M) \subset U C_{w}(X, M)$.

\subsection{The weighted minimal covering set $\left(M C_{\mathrm{w}}\right)$}

We now show that the order of inclusion between the weighted and unweighted minimal covering sets is the same as for the uncovered set.

Proposition 6. $M C\left(X, T^{M}\right) \subseteq M C_{w}(X, M)$ for any 0-weighted tournament $(X, M)$.

Proof. From Lemma 2, we know that $M C_{\mathrm{w}}(X, M)$ is a weak covering set and, from Lemma 3 , that $M C\left(X, T^{M}\right)$ is the unique minimal weak covering set of $(X, M)$. Thus, $M C\left(X, T^{M}\right) \subseteq M C_{\mathrm{w}}(X, M)$.

Remark 5. Example 2 exhibits a voting tournament $(X, M)$ such that $M C\left(X, T^{M}\right) \subset$ $M C_{w}(X, M)$.

Remark 6. By definition, $M C_{w}(X, M) \subseteq \overline{U C}_{w}(X, M) \subseteq U C_{w}(X, M)$. Combining Proposition 6 with $M C \subseteq U C \subseteq T C$ of Remark 1 yields: $M C_{w}(X, M) \cap U C\left(X, T^{M}\right) \neq \emptyset$ and $M C_{w}(X, M) \cap T C\left(X, T^{M}\right) \neq \emptyset$ for any 0 -weighted tournament $(X, M)$.

Remark 7. Example 2 shows that $M C_{w}(X, M) \nsubseteq T C\left(X, T^{M}\right)$ and $T C\left(X, T^{M}\right) \nsubseteq M C_{w}(X, M)$. The same example shows that $M C_{w}(X, M) \nsubseteq U C\left(X, T^{M}\right)$ and $U C\left(X, T^{M}\right) \nsubseteq M C_{w}(X, M)$. 


\subsection{The weighted bipartisan set $\left(B P_{\mathrm{w}}\right)$}

The results of this subsection are established for voting tournaments with off-diagonal odd entries.

Proposition 7. If $(X, M)$ is a voting tournament with odd $m(x, y) \forall x \neq y$, then $B P_{w}(X, M) \subseteq T C_{w}(X, M)$ and $B P_{w}(X, M) \subseteq M C_{w}(X, M)$.

Proof. From a result of Laffond et al. (1997), the game induced by this tournament has a unique Nash equilibrium in mixed strategies and this equilibrium is symmetric. $B P_{\mathrm{w}}(X, M)$ is the support of this equilibrium. Using results in Duggan and Le Breton (1997a), we may assert that if $A_{1} \times A_{2}$ is a WGSP or a MGSP, then $B P_{\mathrm{w}}(X, M) \subseteq A_{1}$ and $B P_{\mathrm{w}}(X, M) \subseteq A_{2}$. This establishes the two parts of the proposition.

Remark 8. Combining Proposition 7 with $M C_{w}(X, M) \subseteq U C_{w}(X, M)$ of Remark 6, we obviously have $B P_{w}(X, M) \subseteq U C_{w}(X, M)$ for any voting tournament with odd $m(x, y)$ $\forall x \neq y$.

Remark 9. Example 2 shows that there exists a voting tournament $(X, M)$ such that $B P_{w}(X, M) \subset T C_{w}(X, M)$ and $B P_{w}(X, M) \subset M C_{w}(X, M)$.

Proposition 8. (a) $T C_{w}(X, M) \cap M C_{w}(X, M) \neq \emptyset$ for any voting tournament $(X, M)$ with odd $m(x, y) \forall x \neq y$.

(b) There exists a voting tournament $(X, M)$ such that $T C_{w}(X, M) \nsubseteq M C_{w}(X, M)$ and $M C_{w}(X, M) \nsubseteq T C_{w}(X, M)$.

Proof. (a) By Proposition 7, both $T C_{\mathrm{w}}(X, M)$ and $M C_{\mathrm{w}}(X, M)$ contain $B P_{\mathrm{w}}(X, M)$.

(b) This follows from Example 2.

Remark 10. Combining Proposition 8 and $M C_{w}(X, M) \subseteq U C_{w}(X, M)$ of Remark 6, we obtain $U C_{w}(X, M) \cap T C_{w}(X, M) \neq \emptyset$ for any voting tournament $(X, M)$ with odd $m(x, y)$ $\forall x \neq y$. Example 2 shows that there exists a voting tournament $(X, M)$ such that $T C_{w}(X, M) \nsubseteq U C_{w}(X, M)$ and $U C_{w}(X, M) \nsubseteq T C_{w}(X, M)$.

Remark 11. The example used in the proof of Proposition 2 shows that there exists a voting tournament $(X, M)$ for which $B P_{w}(X, M) \cap U C\left(X, T^{M}\right)=B P_{w}(X, M) \cap M C\left(X, T^{M}\right)=$ $B P_{w}(X, M) \cap B P\left(X, T^{M}\right)=\emptyset$.

\subsection{The Kemeny set (Ke)}

Proposition 9. There exists a voting tournament $(X, M)$ such that $\operatorname{Ke}(X, M) \cap$ $B P\left(X, T^{M}\right)=\operatorname{Ke}(X, M) \cap M C\left(X, T^{M}\right)=\operatorname{Ke}(X, M) \cap U C\left(X, T^{M}\right)=\emptyset$.

Proof. Consider the following voting tournament: 


\begin{tabular}{lllll}
\hline & $a$ & $b$ & $c$ & $d$ \\
\hline$a$ & 0 & 3 & -1 & -1 \\
$b$ & -3 & 0 & 5 & -1 \\
$c$ & 1 & -5 & 0 & 3 \\
$d$ & 1 & 1 & -3 & 0 \\
\hline
\end{tabular}

It is easy to see that $U C\left(X, T^{M}\right)=\{b, c, d\}$. Using Lemma 4, one can check that $(a, b, c, d)$ is the unique Kemeny order so that $\operatorname{Ke}(X, M)=\{a\}$. Since $B P \subseteq M C \subseteq U C$, we have the result.

Proposition 10. $\operatorname{Ke}(X, M) \subseteq U C_{w}(X, M)$ for any 0-weighted tournament $(X, M)$.

Proof. We prove a stronger claim: $x C_{\mathrm{w}}(M) y \Rightarrow x O y$ for every Kemeny order $O$. Suppose on the contrary that there exists a Kemeny order $O \equiv\left(\ldots, y, x_{1}, x_{2}, x_{3}, \ldots, x_{k}, x, \ldots\right)$ and consider the order $O^{\prime}$ obtained by permuting $x$ and $y$ in $O$. Then,

$$
\begin{aligned}
\sum_{\substack{u \in O^{\prime} v \in O^{\prime} \\
u O^{\prime} v}} \sum_{\substack{u \in v^{\prime} \\
u O v}} m(u, v)-\sum_{i=1} \sum_{i=1} m(u, v)= & \sum_{i=1}^{k}\left(m\left(x, x_{i}\right)-m\left(y, x_{i}\right)\right)+\sum_{i=1}^{k}\left(m\left(x_{i}, y\right)-m\left(x_{i}, x\right)\right) \\
& +m(x, y)-m(y, x)=2 \sum_{i=1}^{k}\left(m\left(x, x_{i}\right)-m\left(y, x_{i}\right)\right) \\
& +m(x, y)-m(y, x) .
\end{aligned}
$$

Since $x C_{\mathrm{w}}(M) y$, the first term on the right-hand side is non negative and the last one is strictly positive. Therefore, by Lemma 4, $O$ is not a Kemeny order, a contradiction.

Remark 12. Examples 1 and 2 both show that there exists a voting tournament $(X, M)$ such that $\operatorname{Ke}(X, M) \subset U C_{w}(X, M)$.

Proposition 11. There exists a voting tournament $(X, M)$ such that $\operatorname{Ke}(X, M) \cap$ $M C_{w}(X, M)=\operatorname{Ke}(X, M) \cap B P_{w}(X, M)=\emptyset$.

Proof. Consider the following voting tournament $(X, M)$, which induces the tournament of Proposition 3.1 in Laffond et al. (1995):

\begin{tabular}{lllllllll}
\hline & $a$ & $b$ & $c$ & $d$ & $e$ & $f$ & $g$ & $h$ \\
\hline$a$ & 0 & 1 & 1 & -1 & -1 & 1 & 1 & 1 \\
$b$ & -1 & 0 & 1 & -1 & -1 & 1 & 1 & 1 \\
$c$ & -1 & -1 & 0 & 1 & 1 & 1 & 1 & 1 \\
$d$ & 1 & 1 & -1 & 0 & 1 & -1 & -1 & -1 \\
$e$ & 1 & 1 & -1 & -1 & 0 & 1 & 1 & 1 \\
$f$ & -1 & -1 & -1 & 1 & -1 & 0 & 1 & 1 \\
$g$ & -1 & -1 & -1 & 1 & -1 & -1 & 0 & 1 \\
$h$ & -1 & -1 & -1 & 1 & -1 & -1 & -1 & 0 \\
\hline
\end{tabular}


Since $S l\left(X, T^{M}\right)=\{e\}$ and $M C\left(X, T^{M}\right)=B P\left(X, T^{M}\right)=U C^{2}\left(X, T^{M}\right)=\{a, b, c\}$, it follows from Laffond et al. (1995) that $K e(X, M)=\{e\}$ and $M C_{\mathrm{w}}(X, M)=B P_{\mathrm{w}}(X, M)=$ $\{a, c, d\}$.

Proposition 12. There exists a voting tournament $(X, M)$ such that $\operatorname{Ke}(X, M) \cap$ $T C_{w}(X, M)=\emptyset$.

Proof. Consider the following voting tournament:

\begin{tabular}{lllllllll}
\hline & $a$ & $b$ & $c$ & $d$ & $e$ & $f$ & $g$ & $h$ \\
\hline$a$ & 0 & 9 & 9 & -9 & -7 & 11 & 11 & 11 \\
$b$ & -9 & 0 & 9 & -9 & -7 & 11 & 11 & 11 \\
$c$ & -9 & -9 & 0 & 9 & 11 & 11 & 11 & 11 \\
$d$ & 9 & 9 & -9 & 0 & 9 & -7 & -7 & -7 \\
$e$ & 7 & 7 & -11 & -9 & 0 & 9 & 9 & 9 \\
$f$ & -11 & -11 & -11 & 7 & -9 & 0 & 9 & 9 \\
$g$ & -11 & -11 & -11 & 7 & -9 & -9 & 0 & 9 \\
$h$ & -11 & -11 & -11 & 7 & -9 & -9 & -9 & 0 \\
\hline
\end{tabular}

It can be checked that $T C_{\mathrm{w}}(X, M)=\{a, b, c, d\}$ and $K e(X, M)=\{e\}$.

3.6. The Simpson-Kramer minmax set (SK)

Proposition 13. There exists a voting tournament $(X, M)$ such that

$$
\begin{aligned}
S K(X, M) & \cap B P_{w}(X, M)=S K(X, M) \cap T C_{w}(X, M)=S K(X, M) \cap B P\left(X, T^{M}\right) \\
& =S K(X, M) \cap M C\left(X, T^{M}\right)=S K(X, M) \cap U C\left(X, T^{M}\right)=S K(X, M) \\
& \cap T C\left(X, T^{M}\right)=S K(X, M) \cap K e(X, M)=\emptyset .
\end{aligned}
$$

Proof. This follows from Example 2 and the fact that $B P \subseteq M C \subseteq U C \subseteq T C$ by Remark 1.

Remark 13. Examples 1 and 2 also show that a Simpson-Kramer winner may be a Condorcet loser, i.e. it can lose in pairwise comparisons against every other alternative.

Proposition 14. (a) $S K(X, M) \cap M C_{w}(X, M) \neq \emptyset$ for any 0 -weighted tournament $(X, M)$.

(b) There exists a voting tournament $(X, M)$ such that $M C_{w}(M) \nsubseteq S K(M)$ and $S K(X, M) \nsubseteq M C_{w}(M)$.

Proof. (a) Suppose $S K(X, M) \cap M C_{\mathrm{w}}(X, M)=\emptyset$ and take any $k \in S K(X, M)$. Since $k \notin M C_{\mathrm{w}}(X, M), \exists a \in M C_{\mathrm{w}}(X, M): a C_{\mathrm{w}}(X, M) k$ relative to $M C_{\mathrm{w}}(X, M) \cup\{k\}$, i.e. $m(a, k)>$ 0 and

$$
m(a, z) \geq m(k, z), \forall z \in M C_{\mathrm{w}}(M) /\{a\} .
$$


We distinguish two cases:

Case 1: $\exists x \in M C_{\mathrm{w}}(X, M) \cap \arg \min _{z \in X /\{a\}} m(a, z)$. By (2), $m(a, z) \geq m(k, z)$. Thus, $a \in$ $S K(X, M)$, a contradiction.

Case 2: $M C_{\mathrm{w}}(X, M) \cap \arg \min _{z \in X /\{a\}} m(a, z)=\emptyset$. Take any $x \in \arg \min _{z \in X /\{a\}} m(a, z)$. Since $x \notin M C_{\mathrm{w}}(X, M), \exists b \in M C_{\mathrm{w}}(X, M): b C_{\mathrm{w}}(M) x$ relative to $M C_{\mathrm{w}}(X, M) \cup\{x\}$. We must have $m(a, x)<0$. Otherwise, $a$ would be a Condorcet winner and one would have $S K(X, M)=M C_{\mathrm{w}}(X, M)=\{a\}$. Since $m(b, x)>0$ and $m(a, k)>0$, we thus have $b \neq a$ and $x \neq k$. From $(2)$ :

$$
m(a, b) \geq m(k, b) .
$$

Moreover, $b C_{\mathrm{w}}(M) x$ relative to $M C_{\mathrm{w}}(X, M) \cup\{x\}$ implies:

$$
m(b, a) \geq m(x, a) .
$$

Combining (3) and (4) yields $m(a, x)=-m(x, a) \geq-m(b, a)=m(a, b) \geq m(k, b)$. Thus, $a \in S K(X, M)$, a contradiction.

(b) Consider the following voting tournament $M$ :

\begin{tabular}{lllll}
\hline & $a$ & $b$ & $c$ & $d$ \\
\hline$a$ & 0 & 1 & -1 & 3 \\
$b$ & -1 & 0 & -1 & -1 \\
$c$ & 1 & 1 & 0 & -3 \\
$d$ & -3 & 1 & 3 & 0 \\
\hline
\end{tabular}

One can easily check that $S K(X, M)=\{a, b\}$ and that $U C_{\mathrm{w}}(X, M)=M C_{\mathrm{w}}(X, M)=$ $\{a, c, d\}$.

Remark 14. Combining Proposition 14 and $M C_{w} \subseteq U C_{w}$ of Remark 6, we get $S K(X, M) \cap U C_{w}(X, M) \neq \emptyset$ for any 0 -weighted tournament $(X, M)$. The voting tournament used in the proof of Proposition 14 gives $U C_{w}(X, M) \nsubseteq S K(X, M)$ and $S K(X, M) \nsubseteq U C_{w}(X, M)$.

\section{Conclusion}

In this article, we have introduced three new solutions for weighted tournaments, which are the weighted equivalents of the Top Cycle, the Uncovered set, and the Minimal Covering set. We have also performed a comparison of 11 solution sets for weighted and unweighted tournaments in terms of inclusion, intersection or absence of the latter.

A first conclusion that can be drawn from these comparisons is that the weighted uncovered set $U C_{\mathrm{w}}$ is a superset of most other solution sets with three exceptions. $U C_{\mathrm{w}}$ always intersects $T C, T C_{\mathrm{w}}$, and $S K$ without being a subset or a superset of the latter. $U C_{\mathrm{w}}$ includes the Borda winners and the Condorcet winner whenever it exists, despite 
the fact that it is not Condorcet consistent. Thus $U C_{\mathrm{w}}$ should rally both Condorcet and Borda advocates as being a set within which the choice of an alternative should be made.

A second conclusion that can be drawn from these comparisons is that the chain of inclusions $B P \subseteq M C \subseteq U C \subseteq T C$ established for tournaments is lost when we move to weighted tournaments. We still obtain $B P_{\mathrm{w}} \subseteq M C_{\mathrm{w}} \subseteq U C_{\mathrm{w}}$ and $B P_{\mathrm{w}} \subseteq T C_{\mathrm{w}}$. However, while $T C_{\mathrm{w}}$ and $U C_{\mathrm{w}}$ always intersect, it can happen that none contains the other. The same is true of $M C_{\mathrm{w}}$ and $T C_{\mathrm{w}}$.

A third conclusion concerns the comparison between tournaments and weighted tournaments. Since weighted tournaments contain more information than tournaments, one could expect solution sets for weighted tournaments to refine their tournament counterparts. Surprisingly, this is the case only for $T C$. Indeed, $T C_{\mathrm{w}} \subseteq T C$ and, in many examples that we worked out, $T C_{\mathrm{w}}$ is much smaller than $T C$. But this order of inclusion is reversed for $U C$ and $M C$ while $B P$ and $B P_{\mathrm{w}}$ might not even intersect.

A final conclusion deals with the position of $K e$ and $S K$ within the family of solutions examined in this article. Since $K e$ and $S K$ are defined without any reference to the solution concepts for the game induced by a weighted tournament, we might expect the absence of clear relations with the solution sets that have a relation with solution concepts for games. This is confirmed by our results. Except for $K e \subseteq T C, K e \subseteq U C_{\mathrm{w}}$, $S K \cap U C_{\mathrm{w}} \neq \emptyset$, and $S K \cap M C_{\mathrm{w}} \neq \emptyset$, the intersection of $K e$ and $S K$ with any of the other solution sets may be empty.

\section{Acknowledgements}

We thank Jean-François Laslier and two anonymous referees for their helpful comments. Philippe De Donder would like to thank the GREQAM-LEQAM for its hospitality, the Belgian Federal Services of Scientific Affairs (SSTC contract SE/13/ 050) and the Communauté française de Belgique for their financial support. Michel Truchon would like to acknowledge the financial support of the Social Sciences and Humanities Research Council of Canada.

\section{References}

Barthelemy, J.P., Guénoche, A., Hudry, O., 1989. Median linear orders: heuristics and a branch and bound algorithm. Eur. J. Operat. Res. 42, 313-325.

Borda, J.C., 1784. Mémoires sur les élections au scrutin, Histoire de l'Académie Royale des Sciences pour 1781. Paris (English Translation by De Grazia, A., 1953. Isis 44).

Condorcet, Marquis de, 1785. Essai sur l'application de l'analyse de probabilité des décisions rendues à la pluralité des voix. Imprimerie royale, Paris.

Copeland, A., 1951. A reasonable social welfare function. Mimeo, University of Michigan Seminar on the Applications of Mathematics to Social Sciences.

Debord, B., 1987. Caractérisation des matrices de préférences nettes et méthodes d'agrégation associées. Math. Sci. Hum. 97, 5-17.

Duggan, J., Le Breton, M., 1996. Dutta's minimal covering set and Shapley's saddles. J. Econ. Theor. 70, $257-265$. 
Duggan, J., Le Breton, M., 1997a. Dominance-based solutions for strategic form games. Mimeo, University of Rochester.

Duggan, J., Le Breton, M., 1997b. Mixed refinements of Shapley's saddles in weak majority tournaments. Mimeo, University of Rochester.

Dutta, B., 1988. Covering sets and a new Condorcet correspondence. J. Econ. Theor. 44, 6-80.

Dutta, B., Laslier, J.F., 1999. Comparison functions and choice correspondences. Social Choice Welfare 16, $513-532$.

Fishburn, P.C., 1977. Condorcet social choice functions. SIAM J. Appl. Math. 33, 469-489.

Fishburn, P.C., 1992. Induced binary probabilities and the linear ordering polytope: a status report. Math. Soc. Sci. 23, 67-80.

Guénoche, A., 1995. How to choose according to partial evaluations. In: Bouchon-Meunier, B. et al. (Ed.), Advances in Intelligent Computing, IPMU'1994, Lecture Notes in Computer Sciences, No. 945, Springer, Berlin, pp. 611-618.

Guénoche, A., Vanderputte-Riboud, B., Denis, J.B., 1994. Selecting varieties using a series of trials and a combinatorial ordering method. Agronomie 14, 363-375.

Kemeny, J., 1959. Mathematics without numbers. Daedalus 88, 571-591.

Kramer, G., 1977. A dynamical model of political equilibrium. J. Econ. Theor. 16, 310-334.

Laffond, G., Laslier, J.-F., Le Breton, M., 1993. The bipartisan set of a tournament game. Games Econ. Behav. 5, 182-201.

Laffond, G., Laslier, J.-F., Le Breton, M., 1994. Social-choice mediators. Am. Econ. Rev. Pap. Proc. 84, $448-453$.

Laffond, G., Laslier, J.-F., Le Breton, M., 1995. Condorcet choice correspondences: a set-theoretical comparison. Math. Soc. Sci. 30, 23-35.

Laffond, G., Laslier, J.-F., Le Breton, M., 1997. A theorem on symmetric two-player zero-sum games. J. Econ. Theor. 72, 426-431.

Laslier, J.F., 1997. Tournament Solutions and Majority Voting, Springer, Berlin.

Le Breton, M., Truchon, M., 1997. A Borda measure for social choice functions. Math. Soc. Sci. 34, $249-272$.

McGarvey, D.C., 1953. A theorem on the construction of voting paradoxes. Econometrica 21, 608-610.

Miller, N.R., 1980. A new solution set for tournament and majority voting: further graph-theoretical approaches to the theory of voting. Am. J. Polit. Sci. 24, 68-96.

Moulin, H., 1986. Choosing from a tournament. Social Choice Welfare 3, 271-296.

Moulin, H., 1988. Condorcet's principle implies the no show paradox. J. Econ. Theor. 45, 53-64.

Owen, G., 1982. Game Theory, 2nd ed., Academic Press, New York.

Peris, J.E., Subiza, B., 1999. Condorcet choice correspondences for weak tournaments. Social Choice Welfare 16, 217-231.

Schwartz, T., 1972. Rationality and the myth of the maximum. Noûs 6, 97-117.

Shapley, L., 1964. Some topics in two-person games. In: Dresher, M., Shapley, L., Tucker, A. (Eds.), Advances in Game Theory, Annals of Mathematics Studies, Vol. 52, pp. 1-28.

Simpson, P., 1969. On defining areas of voter choice: Professor Tullock on stable voting. Q. J. Econ. 83, 478-490.

Slater, P., 1961. Inconsistencies in a schedule of paired comparisons. Biometrica 48, 303-312.

Young, H.P., 1988. Condorcet's theory of voting. Am. Polit. Sci. Rev. 82, 1231-1244.

Young, H.P., Levenglick, A., 1978. A consistent extension of election's principle. SIAM J. Appl. Math. 35, 285-300. 